\title{
Effects of Dietary Lysophosphatidylcholine on Growth Performance and Lipid Metabolism of Juvenile Turbot
}

\author{
Houguo Xu $\mathbb{D},{ }^{1,2}$ Xing Luo, ${ }^{1,3}$ Qingzhu Bi, ${ }^{1}$ Zhendong Wang, ${ }^{4}$ Xiaoxue Meng, ${ }^{1}$ Jinshi Liu, ${ }^{1}$ \\ Mei Duan, ${ }^{1}$ Yuliang Wei $\mathbb{D}^{1,2}$ and Mengqing Liang $\mathbb{D}^{1,2}$ \\ ${ }^{1}$ Yellow Sea Fisheries Research Institute, Chinese Academy of Fishery Sciences, 106 Nanjing Road, Qingdao 266071, China \\ ${ }^{2}$ Laboratory for Marine Fisheries Science and Food Production Processes, Qingdao National Laboratory for Marine Science \\ and Technology, 1 Wenhai Road, Qingdao 266237, China \\ ${ }^{3}$ College of Fisheries and Life Science, Dalian Ocean University, 52 Heishijiao Road, Dalian 116023, China \\ ${ }^{4}$ Weifang Kenon Biological Technology Co., Ltd., 508 Zhujiang West Second Street, Weifang 262737, China \\ Correspondence should be addressed to Mengqing Liang; liangmq@ysfri.ac.cn
}

Received 25 October 2021; Accepted 1 December 2021; Published 31 January 2022

Academic Editor: Zhen-Yu Du

Copyright (c) 2022 Houguo Xu et al. This is an open access article distributed under the Creative Commons Attribution License, which permits unrestricted use, distribution, and reproduction in any medium, provided the original work is properly cited.

\begin{abstract}
A 56-day feeding trial was conducted in a flow-through seawater system to investigate the effects of lysophosphatidylcholine (LPC) on growth performance and lipid metabolism of turbot. Four experimental diets were prepared, differing only in the LPC supplementation, namely, 0 (LPC-0, control), 0.1 (LPC-0.1), 0.25 (LPC-0.25), and 0.5\% (LPC-0.5) of dry matter. Each diet was randomly fed to triplicate tanks. LPC-0.1 and LPC-0.25 led to significantly higher weight gain than the control diet, and the highest weight gain was observed in LPC-0.1. Compared to the control group, the LPC-supplemented groups had higher survival and lower hepatosomatic index and viscerosomatic index. LPC- 0.25 led to significantly lower contents of crude lipid and ash in whole fish. Dietary LPC supplementation led to a basic decrease in the lipid metabolism-related biochemical parameters in serum but had only very minor influence on the fatty acid composition in the liver and subcutaneous tissue around the fin (STF). High LPC levels upregulated the mRNA expression of BSAL and ApoE $\alpha$ in both the liver and STF. In conclusion, dietary LPC supplementation (0.1-0.25\%) enhanced the growth, lowered the lipid accumulation in juvenile turbot, and significantly regulated the lipid metabolism. However, it seldom influenced the fatty acid composition.
\end{abstract}

\section{Introduction}

Emulsifier-like feed additives have been widely used in terrestrial animals [1-6]. In recent years, they are also being more and more frequently used in farmed fish, typically bile acids $[7,8]$. The positive effects of bile acids on growth and lipid metabolism of farmed fish have been reported in a number of studies [9-17], including a recent study of ours with a marine teleost, tiger puffer (Takifugu rubripes) [18]. However, another effective emulsifier, lysophospholipid (mostly lysolecithin), which has been demonstrated to have high efficiency in enhancing dietary lipid utilization in terrestrial animals [19-25], has not been well investigated in farmed fish. Relevant information was available only in small number of fish species such as rainbow trout (Onco- rhynchus mykiss) [26, 27], channel catfish (Ictalurus punctatus) [28], and hybrid tilapia (Oreochromis aureus ot $\times$ Oreochromis niloticus ?) [29]. Even in these limited studies, controversial results, mainly related to whether lysophospholipid is effective or related to the effective lysophospholipid dose, were observed. Therefore, more studies are needed to comprehensively evaluate the efficacy of lysophospholipid in fish diets.

Turbot is a worldwide important aquaculture fish species, which has a low lipid content in the muscle but a relatively high lipid content in the subcutaneous tissue around the fin $[30,31]$. The lipid requirement in this fish species is relatively low (around 10\%) [32-35], lower than other marine carnivorous species such as rockfish (Sebastes schlegeli) [36], tongue sole (Cynoglossus semilaevis) [37], and 
golden pompano (Trachinotus ovatus) [38]. However, use of high-lipid diets is being more and more popular in fish farming practice, in consideration of both the proteinsparing effects of dietary lipid and the pursuit of lipidderived weight gain [39-42]. The use of high-lipid diets and the consequent high lipid accumulation in fish body due to inefficient utilization of excess dietary lipid could have great negative impact on the health status of farmed fish $[43,44]$. Attempts, mostly dietary supplementation of bioactive ingredients which can help fish better utilize dietary lipid, such as carnitine, taurine, and bile acid, have been taken to overcome this negative impact [45-49]. In a recent study of ours with another marine carnivorous species, tiger puffer, dietary supplementation of bile acid has been demonstrated to be able to regulate the hepatic lipid homeostasis when fish were fed high-lipid diets [18]. In the present study, we aimed to comprehensively evaluate the effects of dietary lysophosphatidylcholine on growth performance, lipid accumulation, and fatty acid composition of juvenile turbot fed high-lipid diets, as well as to evaluate the regulation of lipid metabolism by dietary lysophosphatidylcholine at the transcription level. Results of this study could be beneficial to the application of lysophospholipids in fish feeds.

\section{Materials and Methods}

2.1. Experimental Diets. The basal diet used fishmeal, soy protein concentrate, and wheat meal as main protein sources and fish oil and soybean oil as the main lipid sources (Table 1). The control diet contained appr. $44 \%$ protein and $15 \%$ lipid. This lipid level was higher than the optimum lipid level in diets for turbot (around 10\%, [34]) and thus was considered a high lipid level. Graded levels of lysophosphatidylcholine (LPC), namely, $0.10 \%, 0.25 \%$, and $0.5 \%$, were added into the control diet to obtain three experimental diets. The LPC product was supplied by Weifang Kenon Biological Technology Co., Ltd. (Weifang, China). The phosphatidylcholine purity of this soy-derived product was $98 \%$, and the available LPC concentration was $5 \%$. The four diets were designated as LPC-0 (control), LPC-0.1, LPC-0.25, and LPC-0.5, respectively. The diets were made, packed, and stored following the standard procedures in our laboratory [50]. Briefly, a customized single-screw pelleting machine was used to produce pellets of $3.0 \times 3.0 \mathrm{~mm}$. The experimental diets were then oven-dried to have a moisture content of around $7 \%$. Before use, the experimental diets were stored at $-20^{\circ} \mathrm{C}$. The fatty acid profiles of the experimental diets are presented in Table 2.

2.2. Experimental Fish, Feeding Procedure, and Sampling. Juvenile turbots used in the present study were purchased from Huanghai Aquaculture Co. Ltd. (Haiyang, China) and had an average initial body weight of appr. $8 \mathrm{~g}$. Prior to the feeding trial, experimental fish were reared in cylindrical polyethylene tanks (height, $100 \mathrm{~cm}$; diameter, $230 \mathrm{~cm}$; 210 fish in each tank) and fed a commercial diet for 7 days to acclimate to the experimental conditions. Flow-through deep-well seawater was used in the acclimating period and the whole feeding trial period. At the onset of the feeding
TABle 1: Formulation and proximate composition of the experimental diets (\% dry matter).

\begin{tabular}{|c|c|c|c|c|}
\hline Ingredient & $\begin{array}{l}\text { LPC- } \\
0\end{array}$ & $\begin{array}{c}\text { LPC- } \\
0.1\end{array}$ & $\begin{array}{l}\text { LPC- } \\
0.25\end{array}$ & $\begin{array}{c}\text { LPC- } \\
0.5\end{array}$ \\
\hline Fish meal & 40 & 40 & 40 & 40 \\
\hline Soy protein concentrate & 10 & 10 & 10 & 10 \\
\hline Soybean meal & 8 & 8 & 8 & 8 \\
\hline Wheat meal & 21.68 & 21.68 & 21.68 & 21.68 \\
\hline Brewer's yeast & 5 & 5 & 5 & 5 \\
\hline Mineral premix ${ }^{a}$ & 0.5 & 0.5 & 0.5 & 0.5 \\
\hline Vitamin premix ${ }^{\mathrm{a}}$ & 1 & 1 & 1 & 1 \\
\hline Monocalcium phosphate & 1 & 1 & 1 & 1 \\
\hline $\begin{array}{l}\text { L-Ascorbyl-2- } \\
\text { polyphosphate }\end{array}$ & 0.2 & 0.2 & 0.2 & 0.2 \\
\hline Choline chloride & 0.2 & 0.2 & 0.2 & 0.2 \\
\hline Betaine & 0.3 & 0.3 & 0.3 & 0.3 \\
\hline Ethoxyquin & 0.02 & 0.02 & 0.02 & 0.02 \\
\hline Calcium propionic acid & 0.05 & 0.05 & 0.05 & 0.05 \\
\hline Fumaric acid & 0.05 & 0.05 & 0.05 & 0.05 \\
\hline Fish oil & 5.5 & 5.5 & 5.5 & 5.5 \\
\hline Soybean oil & 5.5 & 5.5 & 5.5 & 5.5 \\
\hline Soya lecithin & 1 & 0.9 & 0.75 & 0.5 \\
\hline Lysophosphatidylcholine & 0 & 0.1 & 0.25 & 0.5 \\
\hline \multicolumn{5}{|l|}{ Proximate composition } \\
\hline Moisture & 6.40 & 6.67 & 6.68 & 6.80 \\
\hline Crude protein & 44.13 & 44.01 & 44.02 & 43.72 \\
\hline Crude lipid & 14.86 & 15.86 & 15.31 & 16.34 \\
\hline Ash & 8.05 & 8.01 & 8.00 & 8.08 \\
\hline
\end{tabular}

${ }^{\mathrm{a}}$ Vitamin premix and mineral premix, designed for marine fish, were purchased from Qingdao Master Biotech Co., Ltd, Qingdao, China.

trial, experimental fish were distributed into 12 polyethylene tanks $(200 \mathrm{~L}, 42 \times 72 \times 72 \mathrm{~cm}$ ), each of which was stocked with 30 fish. Each diet was randomly assigned to triplicate tanks. The experimental turbots were hand-fed to apparent satiation two times each day (7:30 and 17:30). The feeding trial lasted for 56 days. Fish were reared under the natural photoperiod and ambient temperature of Haiyang (Shandong province, China). During the feeding trial, the water temperature ranged from 16.2 to $16.6^{\circ} \mathrm{C}$; salinity, 27 29; $\mathrm{pH}, 7.4 \sim 7.9$; and dissolved oxygen, 7.5 8.1 $\mathrm{mg} \mathrm{L}^{-1}$. The tanks were cleaned daily by siphoning out residual feed and feces. Dead fish were taken out when found, and the number and weight of dead fish were recorded every day.

At the end of the feeding trial, after being anesthetized with eugenol $(1: 10,000)$, fish in each tank was bulk weighed, and the number of fish was recorded. After that, 2 randomly selected whole fish were collected from each tank for the analysis of proximate composition. Six more fish per tank were dissected to collect the samples of serum, liver, muscle, and subcutaneous tissue around the fin (STF) for other assays. About $2 \mathrm{~mL}$ blood was collected from each fish. Blood samples were collected from the caudal vein and allowed to clot firstly at room temperature for $2 \mathrm{~h}$ and then at $4^{\circ} \mathrm{C}$ for $6 \mathrm{~h}$. After that, centrifugation $(836 \times \mathrm{g}, 10 \mathrm{~min}$, 
TABLE 2: Fatty acid composition of experimental oils and diets (\% total fatty acid).

\begin{tabular}{|c|c|c|c|c|}
\hline Fatty acid & LPC-0 & LPC-0.1 & LPC-0.25 & LPC- 0.5 \\
\hline C14:0 & 3.56 & 3.69 & 3.53 & 3.66 \\
\hline C16:0 & 22.94 & 23.13 & 23.3 & 23.23 \\
\hline C18:0 & 6.06 & 6.05 & 6.22 & 6.05 \\
\hline C20:0 & 0.52 & 0.5 & 0.52 & 0.49 \\
\hline C22:0 & 0.36 & 0.34 & 0.34 & 0.31 \\
\hline$\sum \mathrm{SFA}$ & 33.43 & 33.82 & 33.88 & 34.24 \\
\hline C16:1 & 3.18 & 3.24 & 3.15 & 3.22 \\
\hline C18:1n-9t & 0.13 & 0.15 & 0.14 & 0.15 \\
\hline $\mathrm{C} 18: 1 \mathrm{n}-9 \mathrm{c}$ & 2.94 & 2.98 & 2.96 & 2.96 \\
\hline C20:1n-9 & 1.65 & 1.65 & 1.67 & 1.64 \\
\hline C22:1n-9 & 0.11 & 0.12 & 0.12 & 0.12 \\
\hline C24:1n-9 & 0.18 & 0.06 & 0.07 & 0.1 \\
\hline$\sum$ MUFA & 8.19 & 8.20 & 8.11 & 8.19 \\
\hline $\mathrm{C} 18: 2 \mathrm{n}-6 \mathrm{t}$ & 0.04 & 0.03 & 0.05 & 0.04 \\
\hline$C 18: 2 n-6 c$ & 36.95 & 37.07 & 37.28 & 37.11 \\
\hline C18:3n-6 & 0.22 & 0.28 & 0.27 & 0.22 \\
\hline$C 20: 2 n-6$ & 0.25 & 0.28 & 0.31 & 0.33 \\
\hline C20:4n-6 & 0.84 & 0.84 & 0.89 & 0.83 \\
\hline$\sum$ n-6PUFA & 38.30 & 38.50 & 38.8 & 38.53 \\
\hline C18:3n-3 & 3.96 & 4.01 & 4.00 & 3.96 \\
\hline C20:5n-3 & 4.76 & 4.78 & 4.86 & 4.76 \\
\hline$C 22: 6 n-3$ & 9.8 & 9.69 & 9.99 & 9.75 \\
\hline$\sum$ n-3PUFA & 18.52 & 18.48 & 18.85 & 18.47 \\
\hline$\sum \mathrm{n}-3 / \sum \mathrm{n}-6$ & 0.48 & 0.48 & 0.49 & 0.48 \\
\hline
\end{tabular}

SFA: saturated fatty acid; MUFA: monounsaturated fatty acid; PUFA: polyunsaturated fatty acid; t: trans-form; c: cis-form.

$\left.4^{\circ} \mathrm{C}\right)$ was conducted and the straw-colored supernatants were collected as serum samples. All the sampling procedures were handled on an ice plate, and all tissue samples collected were frozen immediately with liquid nitrogen and then transferred to $-86^{\circ} \mathrm{C}$. All sampling protocols, as well as fish rearing practices, were reviewed and approved by the Animal Care and Use Committee of the Yellow Sea Fisheries Research Institute.

2.3. Analysis of Proximate Composition, Fatty Acids, and Biochemical Parameters of Serum. The proximate composition analysis of experimental diets and whole fish (two fish per tank) was performed according to the standard methods of AOAC. Samples were oven-dried at $105^{\circ} \mathrm{C}$ to a constant weight for moisture analysis. The whole fish were cut into small pieces before being dried. The dry diets and fish samples were thoroughly ground and subsequently used for the analysis of crude protein, crude lipid, and ash. Crude protein was assayed by measuring nitrogen $(\mathrm{N} \times 6.25)$ using the Kjeldahl method, crude lipid by ether extraction using Soxhlet method, and ash by combustion at $550^{\circ} \mathrm{C}$.

The fatty acid composition was analyzed with gas chromatograph (GC-2010 Pro, Shimadzu, Japan). Fatty acids in freeze-dried samples (two replicates for each diet; a pooled sample of three individual fish per tank) were esterified first with $\mathrm{KOH}$-methanol and then with HCL-methanol, on $72^{\circ} \mathrm{C}$ water bath. Fatty acid methyl esters were extracted with hexane and then separated via gas chromatography with a fused silica capillary column (SHRT-2560, $100 \mathrm{~m} \times 0.25 \mathrm{~mm} \times$ $0.20 \mu \mathrm{m}$, Shimadzu, Japan). The column temperature was programmed to rise from $150^{\circ} \mathrm{C}$ up to $200^{\circ} \mathrm{C}$ at a rate of $15^{\circ} \mathrm{C} \mathrm{min}^{-1}$ and then from $200^{\circ} \mathrm{C}$ to $250^{\circ} \mathrm{C}$ at a rate of $2^{\circ} \mathrm{C} \mathrm{min}^{-1}$. Both the injector and detector temperatures were $250^{\circ} \mathrm{C}$. Results were expressed as the percentage of each fatty acid with respect to total fatty acids (TFA).

The biochemical parameters in serum, namely, triacylglycerol (TG), total bile acid (TBA), total cholesterol (TC), high-density lipoprotein cholesterol (HDL-C), low-density lipoprotein cholesterol (LDL-C), and malondialdehyde (MDA), were analyzed using commercial kits (Nanjing Jiancheng Bioengineering Institute, Nanjing, China) according to the manufacturer's instructions. Three serum samples from individual fish per tank were used in relevant analysis.

2.4. Quantitative Real-Time Polymerase Chain Reaction (qRT-PCR) Analysis. Total RNA in the liver and STF samples was extracted using RNAiso Plus (TaKaRa (Dalian), Dalian, China) and reverse transcribed with PrimeScript ${ }^{\mathrm{TM}}$ RT reagent Kit with gDNA Eraser (TaKaRa) according to the user's manual. Specific primers for the reference genes (EF1 $\alpha$ and $\beta$-actin) and target genes were designed based on the sequences in the GenBank and prepared by TsingKe Biological Technology Co., Ltd. (Qingdao, China) (Table 3). The amplification efficiency for all primers, which was estimated by standard curves based on a 6-step 4-fold dilution series of target template, was within $95 \sim 105 \%$, and the coefficients of linear regression $\left(R^{2}\right)$ were $>0.99$. SYBR ${ }^{\circledR}$ Premix Ex $\mathrm{Taq}^{\mathrm{TM}}$ (TaKaRa Biotechnology (Dalian) Co., Ltd., Dalian, China) was used for the real-time qPCR with a quantitative thermal cycler (Roche LightCycler 96, Basel, Switzerland). The reaction system consists of $2 \mu \mathrm{L}$ cDNA template, $10 \mu \mathrm{L} \mathrm{SYBR}{ }^{\circledR}$ Premix $\operatorname{Ex~Taq~}^{\mathrm{TM}}(2 \times), 0.8 \mu \mathrm{L}$ forward primer $(10 \mu \mathrm{M}), 0.8 \mu \mathrm{L}$ reverse primer $(10 \mu \mathrm{M})$, and $6.4 \mu \mathrm{L}$ sterilized water. The program was as follows: $95^{\circ} \mathrm{C}$ for $5 \mathrm{~min}$ followed by 40 cycles of " $95{ }^{\circ} \mathrm{C}$ for $5 \mathrm{~s}, 55^{\circ} \mathrm{C}$ for $20 \mathrm{~s}, 72{ }^{\circ} \mathrm{C}$ for $10 \mathrm{~s}$." Melting curve analysis $\left(1.85^{\circ} \mathrm{C}\right.$ increment $/ \mathrm{min}$ from $58^{\circ} \mathrm{C}$ to $95^{\circ} \mathrm{C}$ ) was performed after the amplification phase for validation of the sole product. Each sample was run in triplicate. The mRNA expression levels were calculated with the qRT-PCR method: $2^{-\Delta \Delta C t}[51]$.

2.5. Calculation and Statistical Methods. Calculations used in this study include the following: viscerosomatic index VSI ( $\%)=$ wet viscera weight $/$ fish body weight $\times 100$; hepatosomatic index HSI $(\%)=$ wet liver weight/fish body weight $\times 100$; condition factor $=$ body weight $/$ (body length 3 ) $\times 100$; feed conversion ratio = diet consumed/weight gain; feed intake $=$ feed consumed $/($ initial average body weight + final average body weight)/2)/fish number/duration $* 100$.

All percentage data were arcsine transformed prior to analysis. All data were subjected to one-way analysis of variance (ANOVA) in SPSS 16.0 for Windows. The Levene test was performed to test the homogeneity of variance. 
Table 3: Primers used in this work.

\begin{tabular}{|c|c|c|}
\hline Primer & Sequence $\left(5^{\prime}-3^{\prime}\right)$ & GenBank reference \\
\hline $\mathrm{ACAC} \beta-\mathrm{F}$ & AATGCCGTTCCTATTCGTC & XM_035639393 \\
\hline ACAC $\beta-R$ & GAGCCTGTCTGAACATCTCG & \\
\hline FAS-F & GGCAACAACACGGATGGATAC & KC189927.1 \\
\hline FAS-R & CTCGCTTTGATTGACAGAACAC & \\
\hline ACOX1-F & TCCGCTACAGTGTCGTTC & XM_035638508 \\
\hline ACOX1-R & AGTCTCCCTGGCTGATGT & \\
\hline CPT1-F & GCCTTTCAGTTCACCATCACA & XM_035614266.1 \\
\hline CPT1-R & ATGCGGCTGACTCGTTTCTT & \\
\hline DGAT1-F & AGAGCCAAGACAGAAGACG & XM_035619975 \\
\hline DGAT1-R & CATTACTCAGCACCAGCAT & \\
\hline ATGL-F & CGAAAGAGGCAAGAAAGC & XM_035643755 \\
\hline ATGL-R & CGTAGTGAGATACCAGGACGT & \\
\hline HSL-F & GCGTGCCCTGCTCTACTTG & XM_035629599 \\
\hline HSL-R & TCTCGCTGAGGCCACTTTC & \\
\hline DAGL $\alpha-F$ & TCTGGAATGCCTGTAACTC & XM_035628256 \\
\hline DAGL $\alpha-R$ & СТССТCTACTGTCACGGACT & \\
\hline LPL-F & CTCCCACGAACGCTCTAT & KC189937.1 \\
\hline LPL-R & GCGGACCTTGTTGATGTT & \\
\hline HL-F & GGGCTACGACATCAAGAAG & XM_035629475 \\
\hline HL-R & TGAAGGAGATATGGAGGTTT & \\
\hline BSAL like-F & CGCCGTCCTGACATTAGC & XM_035609042 \\
\hline BSAL like-R & AGCCTTGCCCTTCTCCСТ & \\
\hline HMGCR-F & CCACGAGCAATGTTGTCCC & XM_035640341.1 \\
\hline HMGCR-R & TTAGGCATCGCTGGTCTTTT & \\
\hline CYP7A1-F & TCAAATAGCCAGCGGCAAAC & XM_035635553.1 \\
\hline CYP7A1-R & CCATGACAGCTTCGACCCTC & \\
\hline ApoA1 like-F & CAGCCTGGAGCAGAGTGT & XM_035637559 \\
\hline ApoA1 like-R & CCATTTGTTTCACCGAGTT & \\
\hline ApoA4 $\alpha-\mathrm{F}$ & AGGATGCTTTCTGGGACTATGT & XM_035620874 \\
\hline ApoA $4 \alpha-\mathrm{R}$ & GAGGCTGTTCACTTCCTTTCC & \\
\hline ApoB100-F & TCTCACCCTCGGTCTCGG & XM_035617338.1 \\
\hline ApoB100-R & TTCAGGTTTCTCCTCACAACGA & \\
\hline $\mathrm{ApoE} \alpha-\mathrm{F}$ & GGCAGCAGATGGAGAAGT & XM_035620876 \\
\hline $\mathrm{ApoE} \alpha-\mathrm{R}$ & TTCAGCAGGTCGTTCAGG & \\
\hline SREBP1-F & CGATCCGCACTCCAAGT & XM_035615397.1 \\
\hline SREBP1-R & CCGCACTGCCCTGAAT & \\
\hline PPAR $\alpha 1-F$ & CTACTCAAGCCTGGACCTCAACGA & JX975469.1 \\
\hline PPAR $\alpha 1-R$ & TCACTGAAGGGACGCCGCA & \\
\hline PPAR $\alpha 2-\mathrm{F}$ & СССТGATAАСАССТТССТСТTТССС & JX975470.1 \\
\hline PPAR $\alpha 2-\mathrm{R}$ & TGTCTCGGTCGTCTTGATGTCCTG & \\
\hline PPAR $\beta-F$ & ACGGCAAAGGCTTCGTTACC & XM_035643796.1 \\
\hline PPAR $\beta-R$ & CTAATGGCAGCAACAAACAGG & \\
\hline $\operatorname{PPAR} \gamma-\mathrm{F}$ & AAGTGACGGAGTTCGCCAAGA & XM_035631101.1 \\
\hline $\operatorname{PPAR} \gamma-\mathrm{R}$ & GTTCATCAGAGGTGCCATCA & \\
\hline $\mathrm{LXR} \alpha-\mathrm{F}$ & GCGTCATCAAGAGTGCCC & XM_035627821.1 \\
\hline $\mathrm{LXR} \alpha-\mathrm{R}$ & ATCTGATTTGCTCCTCCGAG & \\
\hline $\mathrm{HNF} 4 \alpha-\mathrm{F} 1$ & ATGCTTCTCGGAGGTTCTG & XM_035646017.1 \\
\hline $\mathrm{HNF} 4 \alpha-\mathrm{R} 1$ & GAGGGATTGAGGTTGGCTG & \\
\hline
\end{tabular}


TABLE 3: Continued.

\begin{tabular}{lcc}
\hline Primer & \multicolumn{1}{c}{ Sequence $\left(5^{\prime}-3^{\prime}\right)$} & GenBank reference \\
\hline$\beta$-Actin-F & GTAGGTGATGAAGCCCAGAGCA & MT023044.1 \\
$\beta$-Actin-R & CTGGGTCATCTTCTCCTGT & \\
EF $1 \alpha$-F & TATTAACATCGTGGTCATTGG & KU057926.1 \\
EF $\alpha$-R & CAGGCGTACTTGAAGGAG & \\
\hline
\end{tabular}

TABLE 4: Growth performance and somatic parameters of experimental turbot (mean \pm standard error).

\begin{tabular}{lcccc}
\hline Parameter & LPC-0 & LPC-0.1 & LPC-0.25 & LPC-0.5 \\
\hline Initial body weight (g) & $8.02 \pm 0.02$ & $7.97 \pm 0.01$ & $7.99 \pm 0.03$ & $3.99 \pm 0.03$ \\
Final body weight (g) & $34.65 \pm 1.66$ & $39.35 \pm 0.56$ & $38.63 \pm 1.17$ & $37.48 \pm 0.02$ \\
Survival (\%) & $67.78 \pm 19.28$ & $97.78 \pm 2.22$ & 100 & $93.33 \pm 3.85$ \\
Weight gain (\%) & $332.2 \pm 20.32^{\mathrm{b}}$ & $393.6 \pm 6.98^{\mathrm{a}}$ & $383.7 \pm 16.67^{\mathrm{a}}$ & $369.2 \pm 2.05^{\mathrm{ab}}$ \\
Feed conversion ratio & $0.97 \pm 0.10$ & $0.81 \pm 0.00$ & $0.80 \pm 0.02$ & $0.89 \pm 0.04$ \\
Condition factor & $3.21 \pm 0.18$ & $2.95 \pm 0.09$ & $2.92 \pm 0.06$ & $2.99 \pm 0.17$ \\
HSI (\%) & $2.07 \pm 0.24$ & $1.55 \pm 0.04$ & $1.80 \pm 0.05$ & $2.01 \pm 0.70$ \\
VSI (\%) & $6.95 \pm 0.83$ & $3.91 \pm 0.11$ & $4.62 \pm 0.24$ & $5.33 \pm 1.71$ \\
Feed intake (\%) & $1.83 \pm 0.04$ & $1.89 \pm 0.03$ & $1.88 \pm 0.02$ & $1.88 \pm 0.04$ \\
\hline
\end{tabular}

Data in the same row not sharing the same superscript letter were significantly different $(P<0.05)$.

Significant differences between the means were detected by Tukey's multiple range test. The level of significance was chosen at $P<0.05$. The results were presented as means \pm standard errors of the mean (SEM).

\section{Results}

3.1. Growth Performance, Somatic Index, and Proximate Composition. The survival in the LPC-supplemented groups (>93\%) was higher than that in the control group (67.78\%) (Table 4). The weight gain in groups LPC-0.1 and LPC0.25 was significantly $(P<0.05)$ higher compared to the control group, with the highest value observed in group LPC- 0.1 and an intermediate value observed in group LPC-0.5. Groups LPC-0.1 and LPC- 0.25 had much lower HSI and VSI than the control group. The VSI in LPC-0.1 was only nearly half that of the control group. No significant difference was observed in feed intake among dietary groups.

The crude lipid content in LPC- 0.25 was significantly $(P<0.05)$ lower compared to other groups (Table 5). The ash content in LPC- 0.25 was significantly $(P<0.05)$ lower than that in the control group. No significant difference was observed in moisture and crude protein contents among dietary groups.

\subsection{Lipid Metabolism-Related Biochemical Parameters in the} Serum. The TG concentration in LPC- 0.25 was significantly $(P<0.05)$ lower compared to the control group (Table 6). The lowest TBA concentration was observed in LPC-0.1, and the concentration in LPC- 0.1 and LPC- 0.25 was significantly $(P<0.05)$ lower compared to the control group. The TG concentration in LPC- 0.1 and LPC- 0.5 was significantly $(P<0.05)$ lower compared to the control group. The HDL-C concentration in LPC-supplemented groups was signifi- cantly $(P<0.05)$ lower than that in the control group, but group LPC-0.25 had significantly $(P<0.05)$ higher LDL-C concentration than the control group. No significant difference was observed in MDA content.

3.3. Fatty Acid Composition in the Liver and Subcutaneous Tissue around the Fin (STF). Generally, dietary LPC only had a slight effect on the fatty acid composition. In the liver, LPC-0.1 led to significantly $(P<0.05)$ lower C16:0 content but significantly $(P<0.05)$ higher C20:1n-9 content (Table 7). The liver C22:1n-9 content in groups LPC-0.1 and LPC- 0.25 was significantly $(P<0.05)$ higher compared to the control group and group LPC-0.5.

In the STF, LPC-0.1 had significantly $(P<0.05)$ higher contents of C22:0 and C24:1n-9 than the control group (Table 8).

3.4. Lipid Metabolism-Related Gene Expression in the Liver and Subcutaneous Tissue around the Fin (STF). In the liver, compared to the control group, LPC-0.5 significantly $(P<0.05)$ downregulated the mRNA expression of FAS, DAGL $\alpha$, and CYP7A1 (Figure 1). Compared to the control group and group LPC-0.1, groups LPC- 0.25 and LPC- 0.5 significantly $(P<0.05)$ downregulated the transcription of HMGCR but significantly $(P<0.05)$ upregulated that of BSAL-like. The LPC-supplemented groups showed significantly $(P<0.05)$ lower CPT1 transcription than the control group. Group LPC-0.1 had higher gene expression of ApoA1 than other groups. Group LPC- 0.5 had much higher gene expression of ApoA $4 \alpha$ and $\mathrm{ApoE} \alpha$ than the other groups. No significant difference was observed in expression of other lipid metabolism-related genes among the dietary groups (Table 9). 
TABLE 5: Whole-body proximate composition of experimental turbot ( $\%$ wet weight, mean \pm standard error).

\begin{tabular}{lcccc}
\hline Parameter & LPC-0 & LPC-0.1 & LPC-0.25 & LPC-0.5 \\
\hline Moisture & $76.12 \pm 0.41$ & $75.95 \pm 0.29$ & $76.36 \pm 0.36$ & $75.94 \pm 0.31$ \\
Crude protein & $15.33 \pm 0.10$ & $15.11 \pm 0.17$ & $15.30 \pm 0.16$ & $15.51 \pm 0.12$ \\
Crude lipid & $5.94 \pm 0.25^{\mathrm{a}}$ & $5.94 \pm 0.13^{\mathrm{a}}$ & $5.47 \pm 0.06^{\mathrm{b}}$ & $6.10 \pm 0.13^{\mathrm{a}}$ \\
Ash & $3.47 \pm 0.03^{\mathrm{a}}$ & $3.37 \pm 0.03^{\mathrm{ab}}$ & $3.33 \pm 0.03^{\mathrm{b}}$ & \\
\hline
\end{tabular}

Data in the same row not sharing the same superscript letter were significantly different $(P<0.05)$.

TABLE 6: Lipid metabolism-related biochemical parameters in serum of experimental turbot (mean \pm standard error).

\begin{tabular}{lcccc}
\hline Parameters & LPC-0 & LPC-0.1 & LPC-0.25 & LPC-0.5 \\
\hline TG $(\mathrm{mmol} / \mathrm{L})$ & $1.95 \pm 0.16^{\mathrm{a}}$ & $1.79 \pm 0.13^{\mathrm{ab}}$ & $1.52 \pm 0.10^{\mathrm{b}}$ & $1.57 \pm 0.11^{\mathrm{ab}}$ \\
TBA $(\mu \mathrm{mol} / \mathrm{L})$ & $2.51 \pm 0.07^{\mathrm{a}}$ & $2.31 \pm 0.04^{\mathrm{c}}$ & $2.36 \pm 0.04^{\mathrm{bc}}$ & $2.45 \pm 0.03^{\mathrm{ab}}$ \\
TC $(\mathrm{mmol} / \mathrm{L})$ & $2.46 \pm 0.13^{\mathrm{a}}$ & $2.09 \pm 0.05^{\mathrm{b}}$ & $2.23 \pm 0.11^{\mathrm{ab}}$ & $2.04 \pm 0.07^{\mathrm{b}}$ \\
HDL-C $(\mathrm{mmol} / \mathrm{L})$ & $1.42 \pm 0.07^{\mathrm{a}}$ & $1.08 \pm 0.03^{\mathrm{c}}$ & $1.09 \pm 0.04^{\mathrm{c}}$ & $1.28 \pm 0.04^{\mathrm{b}}$ \\
LDL-C $(\mathrm{mmol} / \mathrm{L})$ & $0.42 \pm 0.04^{\mathrm{b}}$ & $0.50 \pm 0.03^{\mathrm{ab}}$ & $0.60 \pm 0.05^{\mathrm{a}}$ & $0.45 \pm 0.05^{\mathrm{b}}$ \\
MDA $(\mathrm{nmol} / \mathrm{mL})$ & $5.03 \pm 0.81$ & $5.55 \pm 0.49$ & $6.36 \pm 0.60$ & $4.80 \pm 0.33$ \\
\hline
\end{tabular}

Data in the same row not sharing the same superscript letter were significantly different $(P<0.05)$. TG: triacylglycerol; TBA: total bile acid; TC: total cholesterol; HDL-C: high-density lipoprotein cholesterol; LDL-C: low-density lipoprotein cholesterol; MDA: malondialdehyde.

TABLE 7: Liver fatty acid composition of experimental turbot (\% total fatty acid, mean \pm standard error).

\begin{tabular}{|c|c|c|c|c|}
\hline Fatty acid & LPC-0 & LPC-0.1 & LPC- 0.25 & LPC-0.5 \\
\hline C14:0 & $3.22 \pm 0.21$ & $3.21 \pm 0.06$ & $3.29 \pm 0.15$ & $3.39 \pm 0.18$ \\
\hline C16:0 & $15.08 \pm 0.27^{\mathrm{a}}$ & $12.76 \pm 0.28^{\mathrm{b}}$ & $13.72 \pm 0.44^{\mathrm{ab}}$ & $14.15 \pm 0.84^{\mathrm{ab}}$ \\
\hline C18:0 & $2.51 \pm 0.16$ & $2.70 \pm 0.32$ & $2.58 \pm 0.33$ & $2.68 \pm 0.10$ \\
\hline C20:0 & $0.13 \pm 0.00$ & $0.12 \pm 0.01$ & $0.13 \pm 0.01$ & $0.13 \pm 0.00$ \\
\hline C22:0 & $0.14 \pm 0.02$ & $0.12 \pm 0.00$ & $0.12 \pm 0.01$ & $0.13 \pm 0.01$ \\
\hline$\sum$ SFA & $21.08 \pm 0.24^{\mathrm{a}}$ & $18.91 \pm 0.28^{\mathrm{b}}$ & $20.38 \pm 0.04^{\mathrm{b}}$ & $20.47 \pm 0.81^{\mathrm{ab}}$ \\
\hline C16:1n-7 & $3.20 \pm 0.07$ & $3.20 \pm 0.08$ & $3.25 \pm 0.04$ & $3.27 \pm 0.06$ \\
\hline C18:1n-9t & $0.10 \pm 0.01$ & $0.10 \pm 0.01$ & $0.11 \pm 0.01$ & $0.10 \pm 0.01$ \\
\hline C18:1n-9c & $22.15 \pm 0.94$ & $24.28 \pm 1.00$ & $24.62 \pm 1.93$ & $23.37 \pm 0.88$ \\
\hline C20:1n-9 & $1.24 \pm 0.03^{\mathrm{b}}$ & $1.45 \pm 0.03^{\mathrm{a}}$ & $1.11 \pm 0.01^{\mathrm{b}}$ & $1.51 \pm 0.11^{\mathrm{a}}$ \\
\hline C22:1n-9 & $0.27 \pm 0.01^{\mathrm{b}}$ & $0.34 \pm 0.03^{\mathrm{a}}$ & $0.37 \pm 0.01^{\mathrm{a}}$ & $0.28 \pm 0.01^{\mathrm{b}}$ \\
\hline C24:1n-9 & $0.02 \pm 0.00$ & $0.03 \pm 0.00$ & $0.03 \pm 0.01$ & $0.03 \pm 0.00$ \\
\hline$\sum$ MUFA & $27.97 \pm 0.36^{b c}$ & $30.33 \pm 0.36^{\mathrm{a}}$ & $27.55 \pm 0.40^{c}$ & $29.44 \pm 0.45^{\mathrm{ab}}$ \\
\hline$C 18: 2 n-6 t$ & $0.14 \pm 0.00$ & $0.02 \pm 0.00$ & $0.02 \pm 0.00$ & $0.15 \pm 0.01$ \\
\hline$C 18: 2 n-6 c$ & $36.26 \pm 1.64$ & $35.36 \pm 1.65$ & $35.02 \pm 2.59$ & $34.96 \pm 0.87$ \\
\hline C18:3n-6 & $0.22 \pm 0.02$ & $0.21 \pm 0.02$ & $0.21 \pm 0.02$ & $0.20 \pm 0.00$ \\
\hline C20:2n-6 & $2.02 \pm 0.05$ & $2.77 \pm 0.23$ & $2.22 \pm 0.44$ & $2.55 \pm 0.21$ \\
\hline C20:3n-6 & $0.10 \pm 0.01$ & $0.11 \pm 0.00$ & $0.10 \pm 0.01$ & $0.11 \pm 0.01$ \\
\hline C20:4n-6 & $0.68 \pm 0.02$ & $0.62 \pm 0.05$ & $0.60 \pm 0.03$ & $0.66 \pm 0.04$ \\
\hline$\sum$ n-6PUFA & $41.01 \pm 0.21^{\mathrm{a}}$ & $37.64 \pm 0.02^{\mathrm{b}}$ & $40.36 \pm 0.12^{\mathrm{a}}$ & $37.92 \pm 0.29^{\mathrm{b}}$ \\
\hline C18:3n-3 & $2.66 \pm 0.06$ & $2.69 \pm 0.16$ & $2.67 \pm 0.22$ & $2.66 \pm 0.11$ \\
\hline$C 20: 5 n-3$ & $2.42 \pm 0.17$ & $2.23 \pm 0.09$ & $2.31 \pm 0.20$ & $2.36 \pm 0.13$ \\
\hline$C 22: 6 n-3$ & $5.82 \pm 0.74$ & $6.08 \pm 0.36$ & $5.93 \pm 0.32$ & $5.96 \pm 0.58$ \\
\hline$\sum \mathrm{n}$-3PUFA & $10.08 \pm 0.39$ & $10.99 \pm 0.15$ & $10.90 \pm 0.50$ & $10.20 \pm 0.27$ \\
\hline$\sum \mathrm{n}-3 / \sum \mathrm{n}-6$ & $0.25 \pm 0.01^{\mathrm{b}}$ & $0.28 \pm 0.01^{\mathrm{a}}$ & $0.29 \pm 0.01^{\mathrm{a}}$ & $0.26 \pm 0.01^{\mathrm{ab}}$ \\
\hline
\end{tabular}

Data in the same row not sharing the same superscript letter were significantly different $(P<0.05)$. SFA: saturated fatty acid; MUFA: monounsaturated fatty acid; PUFA: polyunsaturated fatty acid; t: trans-form; c: cis-form. 
TABLE 8: Fatty acid composition in the subcutaneous tissue around the fin of experimental turbot (\% total fatty acid, mean \pm standard error).

\begin{tabular}{|c|c|c|c|c|}
\hline Fatty acid & LPC-0 & LPC- 0.1 & LPC-0.25 & LPC-0.5 \\
\hline C14:0 & $3.99 \pm 0.03$ & $3.77 \pm 0.13$ & $3.94 \pm 0.07$ & $3.72 \pm 0.04$ \\
\hline $\mathrm{C} 16: 0$ & $18.52 \pm 0.49$ & $18.61 \pm 0.25$ & $19.51 \pm 0.43$ & $19.44 \pm 0.28$ \\
\hline C18:0 & $4.47 \pm 0.10$ & $4.36 \pm 0.08$ & $4.49 \pm 0.02$ & $4.63 \pm 0.18$ \\
\hline C20:0 & $0.29 \pm 0.01^{\mathrm{b}}$ & $0.31 \pm 0.01^{\mathrm{ab}}$ & $0.31 \pm 0.01^{\mathrm{ab}}$ & $0.33 \pm 0.01^{\mathrm{a}}$ \\
\hline $\mathrm{C} 22: 0$ & $0.05 \pm 0.05^{\mathrm{b}}$ & $0.20 \pm 0.01^{\mathrm{a}}$ & $0.18 \pm 0.00^{\mathrm{ab}}$ & $0.12 \pm 0.06^{\mathrm{ab}}$ \\
\hline$\sum$ SFA & $27.33 \pm 0.57$ & $27.25 \pm 0.35$ & $28.42 \pm 0.52$ & $28.38 \pm 0.35$ \\
\hline C16:1n-7 & $4.27 \pm 0.05$ & $4.09 \pm 0.14$ & $4.25 \pm 0.12$ & $4.22 \pm 0.13$ \\
\hline C18:1n-9t & $0^{\mathrm{b}}$ & $0.09 \pm 0.00^{\mathrm{a}}$ & $0.10 \pm 0.00^{\mathrm{a}}$ & $0.07 \pm 0.03^{\mathrm{ab}}$ \\
\hline C18:1n-9c & $3.99 \pm 0.04$ & $3.83 \pm 0.11$ & $3.90 \pm 0.14$ & $3.92 \pm 0.04$ \\
\hline C20:1n-9 & $2.10 \pm 0.06$ & $2.12 \pm 0.07$ & $2.17 \pm 0.07$ & $2.19 \pm 0.08$ \\
\hline $\mathrm{C} 22: 1 \mathrm{n}-9$ & $0.34 \pm 0.04$ & $0.40 \pm 0.01$ & $0.36 \pm 0.02$ & $0.35 \pm 0.05$ \\
\hline C24:1n-9 & $0.20 \pm 0.01^{\mathrm{b}}$ & $0.24 \pm 0.00^{\mathrm{a}}$ & $0.23 \pm 0.01^{\mathrm{ab}}$ & $0.24 \pm 0.01^{\mathrm{a}}$ \\
\hline$\sum$ MUFA & $10.91 \pm 0.05$ & $10.80 \pm 0.34$ & $11.02 \pm 0.33$ & $11.00 \pm 0.30$ \\
\hline $\mathrm{C} 18: 2 \mathrm{n}-6 \mathrm{t}$ & $0.10 \pm 0.05^{\mathrm{ab}}$ & $0.20 \pm 0.00^{\mathrm{a}}$ & $0.04 \pm 0.00^{\mathrm{b}}$ & $0.18 \pm 0.01^{\mathrm{a}}$ \\
\hline$C 18: 2 n-6 c$ & $36.76 \pm 1.15^{\mathrm{ab}}$ & $37.60 \pm 0.45^{\mathrm{a}}$ & $37.87 \pm 0.51^{\mathrm{a}}$ & $35.15 \pm 0.48^{\mathrm{b}}$ \\
\hline$C 18: 3 n-6$ & $0.20 \pm 0.00^{\mathrm{b}}$ & $0.23 \pm 0.00^{\mathrm{a}}$ & $0.24 \pm 0.01^{\mathrm{a}}$ & $0.22 \pm 0.00^{\mathrm{a}}$ \\
\hline$C 20: 2 n-6$ & $1.52 \pm 0.08$ & $1.57 \pm 0.06$ & $1.38 \pm 0.14$ & $1.42 \pm 0.13$ \\
\hline C20:4n-6 & $0.80 \pm 0.06$ & $0.76 \pm 0.02$ & $0.76 \pm 0.02$ & $0.81 \pm 0.05$ \\
\hline$\sum \mathrm{n}-6 \mathrm{PUFA}$ & $40.61 \pm 0.12^{\mathrm{a}}$ & $40.50 \pm 0.40^{\mathrm{a}}$ & $39.03 \pm 0.51^{\mathrm{ab}}$ & $38.18 \pm 0.55^{\mathrm{b}}$ \\
\hline$C 18: 3 n-3$ & $3.63 \pm 0.17$ & $3.59 \pm 0.05$ & $3.68 \pm 0.06$ & $3.43 \pm 0.04$ \\
\hline$C 20: 5 n-3$ & $5.35 \pm 0.18$ & $5.09 \pm 0.16$ & $5.34 \pm 0.05$ & $5.42 \pm 0.14$ \\
\hline$C 22: 6 n-3$ & $9.60 \pm 0.39$ & $9.57 \pm 0.24$ & $9.64 \pm 0.23$ & $9.61 \pm 0.02$ \\
\hline$\sum \mathrm{n}-3 \mathrm{PUFA}$ & $13.96 \pm 0.63$ & $13.16 \pm 0.29$ & $13.32 \pm 0.29$ & $13.72 \pm 0.65$ \\
\hline$\sum \mathrm{n}-3 / \sum \mathrm{n}-6$ & $0.33 \pm 0.01$ & $0.33 \pm 0.00$ & $0.34 \pm 0.01$ & $0.34 \pm 0.01$ \\
\hline
\end{tabular}

Data in the same row not sharing the same superscript letter were significantly different $(P<0.05)$. SFA: saturated fatty acid; MUFA: monounsaturated fatty acid; PUFA: polyunsaturated fatty acid; t: trans-form; c: cis-form.

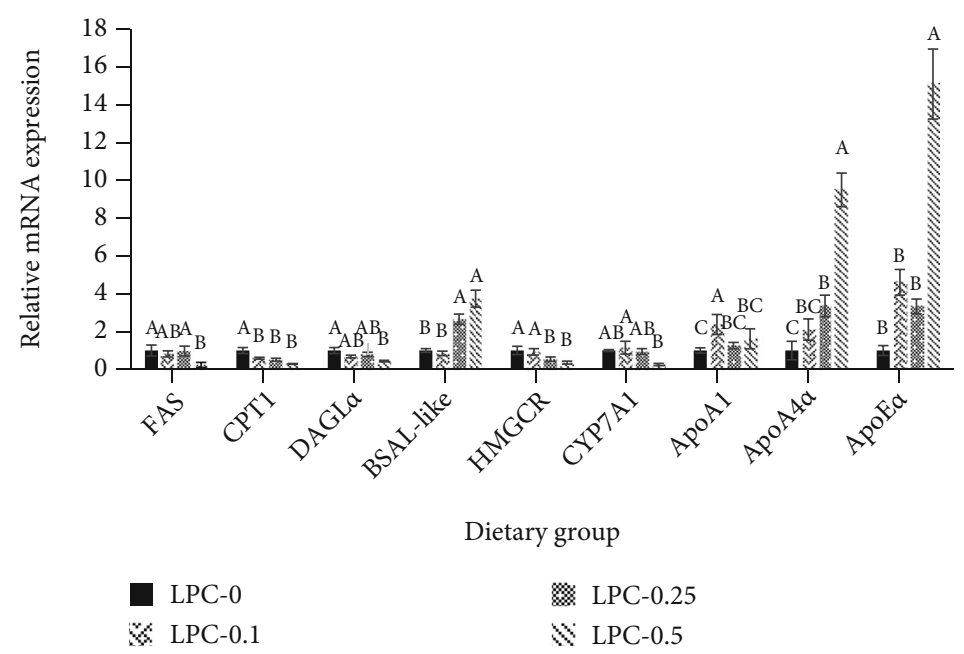

FIGURE 1: Effects of dietary lysophosphatidylcholine supplementation on mRNA expression of lipid metabolism-related genes in the liver of experimental turbot. For a certain gene, data bars not sharing a same superscript letter were significantly different $(P<0.05)$.

In the STF, group LPC-0.5 had much $(P<0.05)$ higher gene expression of BSAL-like, ApoA1, and ApoE $\alpha$ than the other groups (Figure 2). The control group had significantly
$(P<0.05)$ higher gene expression of SREBP1 than groups LPC- 0.25 and LPC-0.5 and significantly $(P<0.05)$ higher gene expression of PPAR $\gamma$ than group LPC-0.25. No 
TABLE 9: Relative mRNA expression of lipid metabolism-related genes in the liver of experimental turbot not showing significant differences among dietary treatments (mean \pm standard error).

\begin{tabular}{lcccc}
\hline Gene & LPC-0 & LPC-0.1 & LPC-0.25 & LPC-0.5 \\
\hline ACAC $\beta$ & $1.00 \pm 0.19$ & $1.00 \pm 0.01$ & $1.12 \pm 0.19$ & $0.88 \pm 0.27$ \\
ACOX1 & $1.00 \pm 0.06$ & $1.53 \pm 0.46$ & $0.94 \pm 0.09$ & $0.83 \pm 0.14$ \\
DGAT1 & $1.00 \pm 0.20$ & $0.82 \pm 0.18$ & $0.91 \pm 0.1$ & $1.33 \pm 0.39$ \\
ATGL & $1.00 \pm 0.39$ & $1.15 \pm 0.17$ & $1.05 \pm 0.13$ & $1.41 \pm 0.39$ \\
HSL & $1.00 \pm 0.09$ & $1.36 \pm 0.44$ & $1.09 \pm 0.22$ & $1.08 \pm 0.26$ \\
LPL & $1.00 \pm 0.24$ & $1.05 \pm 0.32$ & $1.34 \pm 0.21$ & $0.81 \pm 0.15$ \\
HL & $1.00 \pm 0.15$ & $1.54 \pm 0.17$ & $1.55 \pm 0.10$ & $1.38 \pm 0.12$ \\
ApoB100 & $1.00 \pm 0.14$ & $1.40 \pm 0.01$ & $1.20 \pm 0.30$ & $0.79 \pm 0.17$ \\
SREBP1 & $1.00 \pm 0.02$ & $1.12 \pm 0.23$ & $1.23 \pm 0.27$ & $0.9 \pm 0.26$ \\
PPAR $\alpha 1$ & $1.00 \pm 0.01$ & $2.03 \pm 0.81$ & $1.27 \pm 0.40$ & $1.40 \pm 0.60$ \\
PPAR $\alpha 2$ & $1.00 \pm 0.01$ & $1.66 \pm 0.60$ & $1.36 \pm 0.18$ & $1.07 \pm 0.29$ \\
PPAR $\beta$ & $1.00 \pm 0.37$ & $1.41 \pm 0.14$ & $1.27 \pm 0.19$ & $0.97 \pm 0.24$ \\
PPAR $\gamma$ & $1.00 \pm 0.05$ & $1.03 \pm 0.03$ & $1.02 \pm 0.14$ & $0.71 \pm 0.04$ \\
LXR $\alpha$ & $1.00 \pm 0.29$ & $1.29 \pm 0.09$ & $1.17 \pm 0.11$ & $0.73 \pm 0.14$ \\
HNF4 $\alpha$ & $1.00 \pm 0.40$ & $0.77 \pm 0.09$ & $0.90 \pm 0.22$ & $1.02 \pm 0.03$ \\
\hline
\end{tabular}

The expression of all genes listed in this table was not significantly $(P>0.05)$ different among dietary groups.

significant difference was observed in expression of other lipid metabolism-related genes among the dietary groups (Table 10).

\section{Discussion}

The present study demonstrated the significant positive effects of dietary supplementation of LPC (0.1-0.25\%) on growth performance of turbot. This was similar to the study with rainbow trout fed diets containing fat powder, which showed that $0.9 \%$ lysophospholipid in the diet resulted in significant improvement of body weight, specific growth rate, and feed conversion ratio [26]. Another study with rainbow trout also reported significantly improved fish growth, feed intake, and feed utilization by dietary supplementation of $0.3 \%$ soy-derived lysophospholipid [27]. However, a study with channel catfish showed that $0.0125-0.05 \%$ lysolecithin (purity 25\%) did not significantly affect the weight gain of fish [28]. This difference could be due to the low lysolecithin dose used in the later study. It could also be due to the fact that channel catfish is a freshwater fish species and requires a lower lipid level in the diets. Nevertheless, studies with another freshwater species, hybrid tilapia, did not support this explanation [29]. In that study, $0.0125-0.025 \%$ lysophospholipid in the diet significantly improved the growth performance and feed utilization. Since usually all the lysophospholipid products do not have a $100 \%$ purity, other components in the lysophospholipid products may also affect the experimental results. In turbot, there has been a recent study investigating the efficacy of lysolecithin in the diets, which showed that dietary supplementation of $0.1-0.55 \%$ lysolecithin (purity, $20 \%$ ) enhanced the fish growth rate [52]. In that study, a normal dietary lipid level (11\%) was used. Along with the present study using high-lipid diets, it seemed that lysophospholipid is beneficial to growth performance of turbot fed both normal and highlipid diets. In addition, in this study, the survival of turbot was also improved by dietary LPC supplementation. This could be attributed to the improvement of health status, which could be further, at least partly, attributed to lipid metabolism regulation by LPC.

Besides growth-promoting effects, another important function of lysophospholipid was lipid metabolism regulation. In terrestrial animals, lysophospholipid was usually used to enhance the lipid digestion and absorption and thereby to spare dietary lipid [22-25]. This was the typical emulsifying function of lysophospholipid. In fish, however, under the aforementioned background, namely, popular use of high-lipid diets, dietary lysophospholipid was expected to alleviate the negative effects of high-lipid diets. Indeed, this purpose was realized in previous studies on channel catfish [28] and rainbow trout [26, 27], as well as in the present study, which suggested that dietary lysophospholipid reduced the cholesterol and lipid contents in the whole body and liver, which consequently improved the hepatic histology, antioxidation capacity, and immunity. Nevertheless, the regulation of lipid composition by dietary lysophospholipid was not observed in the study with hybrid tilapia [29]. In the former study with turbot, dietary lysolecithin even increased the TG and TC contents in the serum, as well as increased the VSI [52]. These results indicate that lysophospholipid may influence the lipid deposition differently depending on dietary lipid level. In addition, in the present study, although nearly all the lipid-related biochemical parameters in the serum were reduced by dietary LPC, the concentration of LDL-C was increased. This indicates the complexity of LPC function. LDL-C mainly functions as carrier which transports cholesterol biosynthesized in the liver and absorbed from the intestine to peripheral tissues to be utilized $[53,54]$. The increased serum LDL-C content could be related to the deceased basal cholesterol level in fish and thus the increased demand for cholesterol in peripheral tissues. This result was consistent with the downregulated hepatic gene expression of HMGCR, a key enzyme catalyzing the biosynthesis of cholesterol [55], by dietary LPC in the present study.

Regarding the fatty acid composition in experimental fish, dietary LPC exerted a very minor effect on the fatty acid composition in both the liver and STF. The decrease of C16:0 in the liver by dietary LPC could be due to the elevation of $\beta$-oxidation of $\mathrm{C} 16: 0$, but the increase of $\mathrm{C} 20: 1 \mathrm{n}-9$ and C22:1n-9 in the liver, as well the increase of C24:1n-9 in the STF, was difficult to explain based on current information. It has been widely accepted that the fatty acid composition in fish was highly plastic and generally reflected those of the diets [56]. The LPC product used in the present study was soy-derived, and soy lecithin was used to balance the LPC grade in the experimental diets. This resulted in the very similar fatty acid compositions among different diets and probably consequently resulted in very similar fatty acid compositions in fish of different dietary groups. On the other 


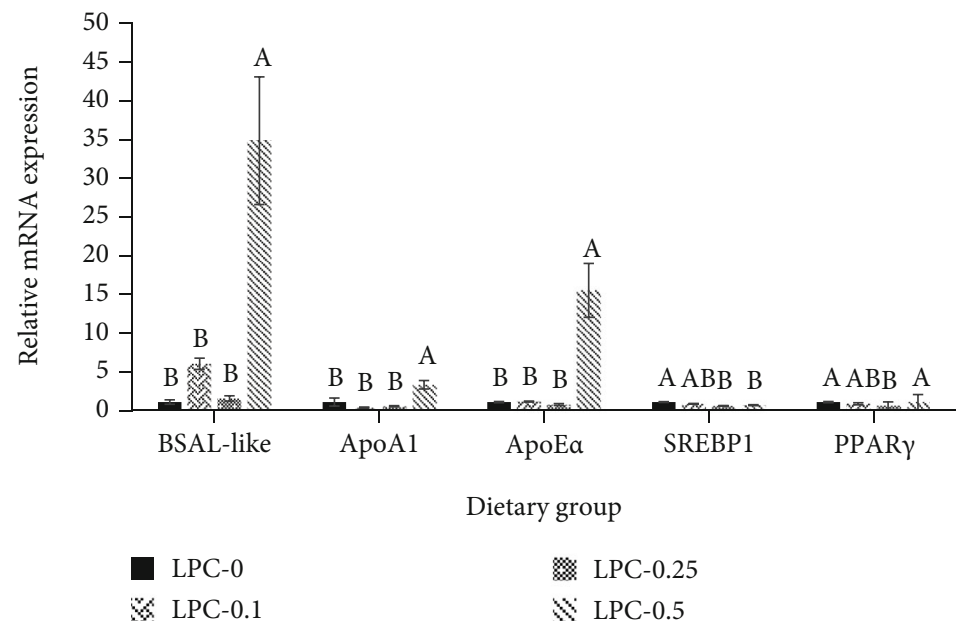

FIGURE 2: Effects of dietary lysophosphatidylcholine supplementation on mRNA expression of lipid metabolism-related genes in the subcutaneous tissue around the fin of experimental turbot. For a certain gene, data bars not sharing the same superscript letter were significantly different $(P<0.05)$.

TABLE 10: Relative mRNA expression of lipid metabolism-related genes in the subcutaneous tissue around the fin of experimental turbot not showing significant differences among dietary treatments (mean \pm standard error).

\begin{tabular}{lcccc}
\hline Gene & LPC-0 & LPC-0.1 & LPC-0.25 & LPC-0.5 \\
\hline ACAC $\beta$ & $1.00 \pm 0.34$ & $0.53 \pm 0.19$ & $0.51 \pm 0.15$ & $0.95 \pm 0.22$ \\
FAS & $1.00 \pm 0.23$ & $0.80 \pm 0.15$ & $0.58 \pm 0.15$ & $0.78 \pm 0.13$ \\
ACOX1 & $1.00 \pm 0.15$ & $0.94 \pm 0.07$ & $0.58 \pm 0.03$ & $0.91 \pm 0.14$ \\
CPT1 & $1.00 \pm 0.20$ & $1.13 \pm 0.18$ & $0.69 \pm 0.09$ & $0.78 \pm 0.07$ \\
DGAT1 & $1.00 \pm 0.13$ & $0.83 \pm 0.10$ & $0.98 \pm 0.12$ & $0.84 \pm 0.13$ \\
ATGL & $1.00 \pm 0.10$ & $0.68 \pm 0.24$ & $0.54 \pm 0.09$ & $0.90 \pm 0.15$ \\
HSL & $1.00 \pm 0.16$ & $0.63 \pm 0.28$ & $0.34 \pm 0.10$ & $0.87 \pm 0.05$ \\
DAGL $\alpha$ & $1.00 \pm 0.23$ & $1.12 \pm 0.31$ & $0.77 \pm 0.09$ & $0.80 \pm 0.02$ \\
LPL & $1.00 \pm 0.18$ & $0.89 \pm 0.23$ & $0.52 \pm 0.04$ & $0.66 \pm 0.16$ \\
HMGCR & $1.00 \pm 0.30$ & $1.31 \pm 0.13$ & $0.98 \pm 0.09$ & $1.28 \pm 0.24$ \\
ApoB100 & $1.00 \pm 0.41$ & $0.61 \pm 0.11$ & $0.36 \pm 0.12$ & $1.03 \pm 0.20$ \\
PPAR $\alpha 1$ & $1.00 \pm 0.24$ & $0.87 \pm 0.08$ & $0.66 \pm 0.10$ & $1.14 \pm 0.15$ \\
PPAR $\alpha 2$ & $1.00 \pm 0.27$ & $0.74 \pm 0.07$ & $0.69 \pm 0.13$ & $0.96 \pm 0.20$ \\
PPAR $\beta$ & $1.00 \pm 0.18$ & $0.99 \pm 0.15$ & $0.64 \pm 0.08$ & $1.01 \pm 0.09$ \\
LXR $\alpha$ & $1.00 \pm 0.14$ & $0.91 \pm 0.23$ & $0.63 \pm 0.09$ & $0.60 \pm 0.01$ \\
HNF4 $\alpha$ & $1.00 \pm 0.04$ & $0.87 \pm 0.08$ & $1.43 \pm 0.21$ & $1.55 \pm 0.30$ \\
\hline
\end{tabular}

The expression of all genes listed in this table was not significantly $(P>0.05)$ different among dietary groups.

hand, the minor difference in fatty acid composition among the experimental diets confirmed that the dietary effects in the present study were attributed to molecular structure of LPC rather than its fatty acid composition.

The effects of dietary LPC on expression of lipid metabolism-related genes in the liver and STF were somehow unexpected. On the one hand, the effects were only moderate. Only 9 out of 25 genes in the liver and even less (5 out of 22) in the STF were significantly affected. After feeding for 56 days, some gene expression possibly had adapted to the experimental diets. On the other hand, among the limited genes significantly regulated by LPC, many were significantly downregulated, including both lipogenic genes such as FAS, HMGCR, CYP7A1, SREBP1, and PPAR $\gamma$ and lipolytic genes such as CPT1 and DAGL $\alpha$. This may be due to the fact that the already decreased basal lipid level by dietary LPC resulted in a less active lipid metabolism. Another characteristic of the gene expression result was that the high LPC dose (0.5\%) had a more significant effect on gene expression than the low LPC doses $(0.1 \%$ and $0.25 \%)$. The aforementioned same reason may explain this characteristic too, because the lipid accumulation seemed less changed in group LPC-0.5.

The most significantly affected two genes in both the liver and STF were BSAL and ApoE $\alpha$. BSAL catalyzes the hydrolysis of a wide range of substrates including cholesteryl esters, phospholipids, lysophospholipids, di- and triacylglycerols, and fatty acid esters of hydroxy fatty acids and thus plays an essential role in the complete digestion of dietary lipids and their intestinal absorption $[57,58]$. ApoE $\alpha$ mediates the transport and uptake of cholesterol and lipid by way of its high affinity interaction with different cellular receptors $[59,60]$. The gene expression of ApoA1 in the liver and STF, as well as that of ApoA4 $\alpha$ in the liver, was also upregulated by LPC supplementation. ApoA1 is the major apolipoprotein of plasma high-density lipoproteins (HDL) and the preferential receptor of phospholipid and free cholesterol, and ApoA4 acts primarily in intestinal lipid absorption $[61,62]$. All these gene expression results evidenced the significant improvement of lipid digestion by dietary LPC, as observed in other fish studies $[26,28,52]$, as well as in studies with terrestrial animals [22-25].

In conclusion, the present study suggested that dietary LPC supplementation (0.1-0.25\%, purity 5\%) enhanced the growth and lowered the lipid accumulation in juvenile turbot. Dietary LPC significantly upregulated the gene expression of BSAL and ApoE in both the liver and the 
subcutaneous tissue around the fin, indicating the enhancement of lipid digestion by LPC. The fatty acid analysis of diets and fish tissues suggested that the effects of dietary LPC could be attributed to the molecular structure of LPC rather than its fatty acid composition.

\section{Abbreviations}

ACAC $\beta$ : Acetyl-CoA carboxylase beta

FAS: $\quad$ Fatty acid synthase

ACOX1: Acyl-CoA oxidase 1, palmitoyl

CPT1: Carnitine O-palmitoyltransferase-1

DGAT1: Diacylglycerol O-acyltransferase 1

ATGL: Adipose triacylglyceride lipase

HSL: Hormone-sensitive lipase

DAGL $\alpha$ : Diacylglycerol lipase, alpha

LPL: $\quad$ Lipoprotein lipase

HL: $\quad$ Hepatic lipase

BSAL: Bile salt activated lipase

HMGCR: 3-Hydroxy-3-Methylglutaryl CoA reductase

CYP7A1: Cholesterol $7 \alpha$-hydroxylase

Apo: Apolipoprotein

SREBP1: Sterol regulatory element binding transcription protein 1

PPAR: Peroxisome proliferators-activated receptor

LXR $\alpha$ : Liver X receptor alpha (nuclear receptor subfamily 1, group $\mathrm{H}$, member 3, NR1H3)

$\mathrm{HNF} 4 \alpha$ : Hepatocyte nuclear factor 4, alpha.

\section{Data Availability}

Raw data supporting the conclusions of this manuscript will be made available by the authors, without undue reservation, to any qualified researcher.

\section{Conflicts of Interest}

The authors declare that they have no conflicts of interest.

\section{Authors' Contributions}

Houguo $\mathrm{Xu}$ and Xing Luo contributed equally to this work.

\section{Acknowledgments}

This work was supported by National Key R\&D Program of China (2018YFD0900400); National Natural Science Foundation of China (31772862); Central Public-interest Scientific Institution Basal Research Fund, CAFS (2020TD48); and China Agriculture Research System of Ministry of Finance (MOF) and Ministry of Agriculture and Rural Affairs (MARA) (CARS-47).

\section{References}

[1] J. Kamran, S. Mehmood, and A. Mahmud, "Effect of fat sources and emulsifier levels in broiler diets on performance, nutrient digestibility, and carcass parameters," The Journal of Poultry Science, vol. 22, no. 1, 2020.
[2] J. H. Park, J. S. Jeong, and I. H. Kim, "Effects of lysophospholipid emulsifier supplementation in the diet of sows," Animal Nutrition and Feed Technology, vol. 17, no. 1, pp. 87-93, 2017.

[3] K. M. Santos, P. H. Watanabe, E. R. Freitas et al., "Beef tallow and emulsifier in growing-finishing pig diets," Anais da Academia Brasileira de Ciências, vol. 89, no. 2, pp. 1221-1230, 2017.

[4] H. Y. Sun and I. H. Kim, "Evaluation of an emulsifier blend on growth performance, nutrient digestibility, blood lipid profiles, and fecal microbial in growing pigs fed low energy density diet," Livestock Science, vol. 227, pp. 55-59, 2019.

[5] A. A. Saleh, K. A. Amber, M. M. Mousa et al., "A mixture of exogenous emulsifiers increased the acceptance of broilers to low energy diets: growth performance, blood chemistry, and fatty acids traits," Animals, vol. 10, no. 3, p. 437, 2020.

[6] S. S. Wickramasuriya, S. P. Macelline, C. H. Min, J. S. Hong, S. H. Park, and J. M. Heo, "Physiological effects of a tallowincorporated diet supplemented with an emulsifier and microbial lipases on broiler chickens," Frontiers in Veterinary Science, vol. 7, p. 583998, 2020.

[7] A. M. Bergman, J. T. Trushenski, and M. Drawbridge, "Addition of emulsifiers to hydrogenated soybean oil-based feeds for yellowtail," North American Journal of Aquaculture, vol. 80, no. 1, pp. 13-23, 2018.

[8] N. Medagoda, M. G. Kim, B. E. Gunathilaka, S. H. Park, and K. J. Lee, "Effect of total replacement of fish oil with tallow and emulsifier in diet on growth, feed utilization, and immunity of olive flounder (Paralichthys olivaceus)," Journal of the World Aquaculture Society, vol. 5, pp. 1-14, 2021.

[9] B. Adhami, A. K. Amirkolaie, H. Oraji, and R. E. Kenari, "Growth performance, nutrient digestibility and lipase activity in juvenile rainbow trout (Oncorhynchus mykiss) fed fat powder in diet containing emulsifiers (cholic acid and Tween80)," Aquaculture Nutrition, vol. 23, no. 5, pp. 1153-1159, 2017.

[10] J. Du, H. Xu, S. Li, Z. Cai, K. Mai, and Q. Ai, "Effects of dietary chenodeoxycholic acid on growth performance, body composition and related gene expression in large yellow croaker (Larimichthys crocea) fed diets with high replacement of fish oil with soybean oil," Aquaculture, vol. 479, pp. 584-590, 2017.

[11] M. Gu, N. Bai, and T. M. Kortner, "Taurocholate supplementation attenuates the changes in growth performance, feed utilization, lipid digestion, liver abnormality and sterol metabolism in turbot (Scophthalmus maximus) fed high level of plant protein," Aquaculture, vol. 468, pp. 597-604, 2017.

[12] Y. Iwashita, N. Suzuki, T. Yamamoto et al., "Supplemental effect of cholyltaurine and soybean lecithin to a soybean meal-based fish meal-free diet on hepatic and intestinal morphology of rainbow troutOncorhynchus mykiss," Fisheries Science, vol. 74, no. 5, pp. 1083-1095, 2008.

[13] Y. Iwashita, N. Suzuki, H. Matsunari, T. Sugita, and T. Yamamoto, "Influence of soya saponin, soya lectin, and cholyltaurine supplemented to a casein-based semipurified diet on intestinal morphology and biliary bile status in fingerling rainbow trout Oncorhynchus mykiss," Fisheries Science, vol. 75, no. 5, pp. 1307-1315, 2009.

[14] M. Jiang, H. Wen, G. W. Gou, T. L. Liu, X. Lu, and D. F. Deng, "Preliminary study to evaluate the effects of dietary bile acids on growth performance and lipid metabolism of juvenile genetically improved farmed tilapia (Oreochromis niloticus) fed plant ingredient-based diets," Aquaculture Nutrition, vol. 24, no. 4, pp. 1175-1183, 2018. 
[15] M. Jin, T. Pan, X. Cheng et al., "Effects of supplemental dietary l-carnitine and bile acids on growth performance, antioxidant and immune ability, histopathological changes and inflammatory response in juvenile black seabream (Acanthopagrus schlegelii) fed high-fat diet," Aquaculture, vol. 504, pp. 199209, 2019.

[16] T. Yamamoto, N. Suzuki, H. Furuita, T. Sugita, N. Tanaka, and T. Goto, "Supplemental effect of bile salts to soybean mealbased diet on growth and feed utilization of rainbow trout Oncorhynchus mykiss," Fisheries Science, vol. 73, no. 1, pp. 123-131, 2007.

[17] J. S. Zhou, H. J. Chen, H. Ji et al., "Effect of dietary bile acids on growth, body composition, lipid metabolism and microbiota in grass carp (Ctenopharyngodon idella)," Aquaculture Nutrition, vol. 24, no. 2, pp. 802-813, 2018.

[18] Z. Liao, B. Sun, Q. Zhang et al., "Dietary bile acids regulate the hepatic lipid homeostasis in tiger puffer fed normal or high-lipid diets," Aquaculture, vol. 519, no. 5, p. 734935, 2020.

[19] W. Boontiam, B. Jung, and Y. Y. Kim, "Effects of lysophospholipid supplementation to lower nutrient diets on growth performance, intestinal morphology, and blood metabolites in broiler chickens," Poultry Science, vol. 96, no. 3, pp. 593-601, 2017.

[20] D. L. Brautigan, R. Li, E. Kubicka et al., "Lysolecithin as feed additive enhances collagen expression and villus length in the jejunum of broiler chickens," Poultry Science, vol. 96, no. 8, pp. 2889-2898, 2017.

[21] C. Chen, B. Jung, and W. K. Kim, "Effects of lysophospholipid on growth performance, carcass yield, intestinal development, and bone quality in broilers," Poultry Science, vol. 98, no. 9, pp. 3902-3913, 2019.

[22] S. M. Hosseini, R. Nourmohammadi, H. Nazarizadeh, and J. D. Latshaw, "Effects of lysolecithin and xylanase supplementation on the growth performance, nutrient digestibility and lipogenic gene expression in broilers fed low-energy wheatbased diets," Journal of Animal Physiology and Animal Nutrition, vol. 102, no. 6, pp. 1564-1573, 2018.

[23] P. Y. Zhao, H. L. Li, M. M. Hossain, and I. H. Kim, "Effect of emulsifier (lysophospholipids) on growth performance, nutrient digestibility and blood profile in weanling pigs," Animal Feed Science and Technology, vol. 207, pp. 190-195, 2015.

[24] P. Y. Zhao, Z. F. Zhang, R. X. Lan, W. C. Liu, and I. H. Kim, "Effect of lysophospholipids in diets differing in fat contents on growth performance, nutrient digestibility, milk composition and litter performance of lactating sows," Animal, vol. 11, no. 6, pp. 984-990, 2017.

[25] M. Zampiga, A. Meluzzi, and F. Sirri, "Effect of dietary supplementation of lysophospholipids on productive performance, nutrient digestibility and carcass quality traits of broiler chickens," Italian Journal of Animal Science, vol. 15, no. 3, pp. 521-528, 2016.

[26] B. Adhami, A. K. Amirkolaei, H. Oraji, M. Kazemifard, and S. Mahjoub, "Effects of lysophospholipid on rainbow trout (Oncorhynchus mykiss) growth, biochemical indices, nutrient digestibility and liver histomorphometry when fed fat powder diet," Aquaculture Nutrition, vol. 27, no. 6, pp. 1779-1788, 2021.

[27] M. Taghavizadeh, S. Shekarabi, M. S. Mehrgan, and H. R. Islami, "Efficacy of dietary lysophospholipids (Lipidol ${ }^{\mathrm{TM}}$ ) on growth performance, serum immuno-biochemical parameters, and the expression of immune and antioxidant- related genes in rainbow trout (Oncorhynchus mykiss)," Aquaculture, vol. 525, p. 735315, 2020.

[28] G. Liu, S. Ma, F. Chen, W. Gao, W. Zhang, and K. Mai, "Effects of dietary lysolecithin on growth performance, feed utilization, intestinal morphology and metabolic responses of channel catfish (Ictalurus punctatus)," Journal of International Management, vol. 26, no. 2, pp. 456-465, 2020.

[29] H. Li, L. Tian, Y. Wang, and Y. Hu, "Effects of lysolecithin on growth performance, body composition and hematological indices of hybrid tilapia (Oreochromisaureus $\overrightarrow{0} \times$ Oreochromisniloticus ()," Journal of Dalian Fisheries University, vol. 25, no. 2, pp. 143-146, 2010.

[30] S. P. Aubourg, V. Losada, and R. Prego, "Distribution of lipids and trace minerals in different muscle sites of farmed and wild turbot (Psetta maxima)," International Journal of Food Science and Technology, vol. 42, no. 12, pp. 1456-1464, 2007.

[31] H. Xu, Q. Bi, E. Pribytkova et al., "Different lipid scenarios in three lean marine teleosts having different lipid storage patterns," Aquaculture, vol. 536, p. 736448, 2021.

[32] S. H. Cho, S. M. Lee, S. M. Lee, and J. H. Lee, "Effect of dietary protein and lipid levels on growth and body composition of juvenile turbot (Scophthalmus maximus L) reared under optimum salinity and temperature conditions," Aquaculture Nutrition, vol. 11, no. 4, pp. 235-240, 2005.

[33] X. Liu, K. Mai, Z. Liufu, and Q. Ai, "Effects of dietary protein and lipid levels on growth, nutrient utilization, and the whole-body composition of turbot, Scophthalmus maximus, Linnaeus 1758, at different growth stages," Journal of the World Aquaculture Society, vol. 45, no. 4, pp. 355-366, 2014.

[34] C. Regost, J. Arzel, M. Cardinal, J. Robin, M. Laroche, and S. J. Kaushik, "Dietary lipid level, hepatic lipogenesis and flesh quality in turbot (Psetta maxima)," Aquaculture, vol. 193, no. 3-4, pp. 291-309, 2001.

[35] H. Sevgili, A. Kurtoglu, M. Oikawa et al., "High dietary lipids elevate carbon loss without sparing protein in adequate protein-fed juvenile turbot (Psetta maxima)," Aquaculture International, vol. 22, no. 2, pp. 797-810, 2014.

[36] S. M. Lee, I. G. Jeon, and J. Y. Lee, "Effects of digestible protein and lipid levels in practical diets on growth, protein utilization and body composition of juvenile rockfish (Sebastes schlegeli)," Aquaculture-Amsterdam, vol. 211, no. 1-4, pp. 227239, 2002.

[37] X. Liu, K. Mai, Q. Ai, X. Wang, Z. Liufu, and Y. Zhang, "Effects of protein and lipid levels in practical diets on growth and body composition of tongue sole,Cynoglossus semilaevis Gunther," Journal of the World Aquaculture Society, vol. 44, no. 1, pp. 96-104, 2013.

[38] X. Ren, M. Zhu, Y. B. Wu et al., "The optimal dietary lipid level for golden pompanoTrachinotus ovatusfed the diets with fish meal replaced by soy protein concentrate," Aquaculture Research, vol. 52, no. 7, pp. 3350-3359, 2021.

[39] X. Li, Y. Jiang, W. Liu, and X. Ge, "Protein-sparing effect of dietary lipid in practical diets for blunt snout bream (Megalobrama amblycephala) fingerlings: effects on digestive and metabolic responses," Fish Physiology and Biochemistry, vol. 38, no. 2, pp. 529-541, 2012.

[40] S. Morais, J. G. Bell, D. A. Robertson, W. J. Roy, and P. C. Morris, "Protein/lipid ratios in extruded diets for Atlantic cod ( _Gadus morhua_ L.): effects on growth, feed utilisation, muscle composition and liver histology," Aquaculture, vol. 203, no. 1-2, pp. 101-119, 2001. 
[41] L. Wang, W. R. Zhang, S. Gladstone, W. K. Ng, J. Z. Zhang, and Q. J. Shao, "Effects of isoenergetic diets with varying protein and lipid levels on the growth, feed utilization, metabolic enzymes activities, antioxidative status and serum biochemical parameters of black sea bream (Acanthopagrus schlegelii)," Aquaculture, vol. 513, p. 734397, 2019.

[42] M. Yigit, Ö. Yardim, and S. Koshio, "Spatio-temporal analysis of commercial trawler data using general additive models: patterns of Loliginid squid abundance in the north-east Atlantic," Israeli Journal of Aquaculture - Bamidgeh, vol. 59, no. 3, pp. 633-648, 2002.

[43] Z. Y. Du, P. Clouet, W. H. Zheng, P. Degrace, L. X. Tian, and Y. J. Liu, "Biochemical hepatic alterations and body lipid composition in the herbivorous grass carp (Ctenopharyngodon idella) fed high-fat diets," British Journal of Nutrition, vol. 95, no. 5, pp. 905-915, 2006.

[44] K. L. Lu, W. N. Xu, X. F. Li, W. B. Liu, L. N. Wang, and C. N. Zhang, "Hepatic triacylglycerol secretion, lipid transport and tissue lipid uptake in blunt snout bream (Megalobrama amblycephala) fed high-fat diet," Aquaculture, vol. 408-409, pp. 160168, 2013.

[45] C. T. Kalinowski, J. Socorro, and L. E. Robaina, "Effect of dietary canthaxanthin on the growth and lipid composition of red porgy (Pagrus pagrus)," Aquaculture Research, vol. 46, no. 4, pp. 893-900, 2015.

[46] Z. Liao, H. Xu, Y. Wei, Q. Zhang, and M. Liang, "Dietary astaxanthin differentially affected the lipid accumulation in the liver and muscle of the marine teleost, tiger pufferTakifugu rubripes," Aquaculture Research, vol. 49, no. 10, pp. 34213433, 2018.

[47] H. Xu, L. Cao, Y. Wei, Y. Zhang, and M. Liang, "Lipid contents in farmed fish are influenced by dietary DHA/EPA ratio: a study with the marine flatfish, tongue sole (Cynoglossus semilaevis)," Aquaculture Amsterdam, vol. 485, pp. 183-190, 2018.

[48] H. Xu, Q. Zhang, Y. Wei, Z. Liao, and M. Liang, "Dietary methionine increased the lipid accumulation in juvenile tiger puffer_Takifugu rubripes_," Part B. Biochemistry \& Molecular Biology, vol. 230, pp. 19-28, 2019.

[49] H. Xu, Q. Zhang, S. K. Kim et al., "Dietary taurine stimulates the hepatic biosynthesis of both bile acids and cholesterol in the marine teleost, tiger puffer (Takifugu rubripes)," British Journal of Nutrition, vol. 123, no. 12, pp. 1345-1356, 2020.

[50] H. Xu, Y. Mu, Y. Zhang et al., "Graded levels of fish protein hydrolysate in high plant diets for turbot (Scophthalmus maximus): effects on growth performance and lipid accumulation," Aquaculture, vol. 454, pp. 140-147, 2016.

[51] K. J. Livak and T. D. Schmittgen, "Analysis of relative gene expression data using real-time quantitative PCR and the $2^{-\Delta \Delta_{-} C_{-}}$method," Methods, vol. 25, no. 4, pp. 402-408, 2001.

[52] L. Y. Li, S. M. Limbu, Q. Ma, L. Q. Chen, M. L. Zhang, and Z. Y. $\mathrm{Du}$, "The metabolic regulation of dietary L-carnitine in aquaculture nutrition: present status and future research strategies," Reviews in Aquaculture, vol. 11, no. 4, pp. 1228-1257, 2019.

[53] A. Pirillo, A. L. Catapano, and G. D. Norata, "Recent insights into low-density lipoprotein metabolism and therapy," Current Opinion in Clinical Nutrition and Metabolic Care, vol. 24, no. 2, pp. 120-126, 2021.

[54] D. P. Sherbet, P. Garg, E. S. Brilakis, and S. Banerjee, "Lowdensity lipoprotein cholesterol: how low can we go?," American Journal of Cardiovascular Drugs, vol. 13, no. 4, pp. 225$232,2013$.
[55] K. L. Luskey and B. Stevens, "Human 3-hydroxy-3-methylglutaryl coenzyme A reductase. Conserved domains responsible for catalytic activity and sterol-regulated degradation.," Journal of Biological Chemistry, vol. 260, no. 18, pp. 1027110277, 1985.

[56] H. Xu, G. M. Turchini, D. S. Francis et al., "Are fish what they eat? A fatty acid's perspective," Progress in Lipid Research, vol. 80, p. 101064, 2020.

[57] H. Tanaka, I. Mierau, and F. Ito, "Purification and characterization of bovine pancreatic bile salt-activated lipase," The Journal of Biochemistry, vol. 125, no. 5, pp. 883-890, 1999.

[58] X. Xiao, G. Jones, W. A. Sevilla et al., "A carboxyl ester lipase (CEL) mutant causes chronic pancreatitis by forming intracellular aggregates that activate apoptosis," The Journal of Biological Chemistry, vol. 291, no. 44, pp. 23224-23236, 2016.

[59] J. R. Gee, Q. Ding, and J. N. Keller, "Modulation of apolipoprotein $\mathrm{E}$ and interleukin- $1 \beta$ in the aging liver," Experimental Gerontology, vol. 40, no. 5, pp. 409-415, 2005.

[60] B. S. Kamalam, S. Panserat, P. Aguirre, I. Geurden, S. Fontagné-Dicharry, and F. Médale, "Selection for high muscle fat in rainbow trout induces potentially higher chylomicron synthesis and PUFA biosynthesis in the intestine," Comparative Biochemistry and Physiology. Part A, Molecular \& Integrative Physiology, vol. 164, no. 2, pp. 417-427, 2013.

[61] M. J. Chapman, "[3] Comparative analysis of mammalian plasma lipoproteins," Methods in Enzymology, vol. 128, pp. 70-143, 1986.

[62] H. Wang, J. Du, S. Lu, Y. Yao, F. Hunter, and D. D. Black, "Regulation of intestinal apolipoprotein A-I synthesis by dietary phosphatidylcholine in newborn swine," Lipids, vol. 36, no. 7, pp. 683-687, 2001. 